\title{
VIABILIDADE ECONÔMICA DOS SISTEMAS MANUAL E MECANIZADO DE APLICAÇÃO DE HERBICIDAS EM FERROVIAS'
}

\author{
ULISSES R. ANTUNIASSI ${ }^{2}$, EDIVALDO D. VELINI ${ }^{3}$, DANIEL CAMPOSILVAN ${ }^{4}$
}

\section{RESUMO}

A aplicação mecanizada de herbicidas em ferrovias tem como principal vantagem a grande capacidade operacional, cobrindo trechos extensos em curtos espaços de tempo. Entretanto, em função das características dos equipamentos utilizados, quase sempre ocorre grande desperdício de herbicida. A aplicação manual, através de pulverizadores costais, tem como principal vantagem a possibilidade da pulve rização di rigida às áreas infestad as, diminuindo a quantidade de herbicida aplicado. Por outro lado, sua reduzida capacidade operacional faz com que a aplicação de longos trechos seja demorada, tornando-a, assim, mais dependente dos problemas climáticos e operacionais da ferrovia. O objetivo do trabalho foi realizar uma análise comparativa das características operacionais e econômicas destes sistemas de aplicação, apontando os fatores mais importantes a serem avaliados para a sua implantação. Para tanto, foram realizadas simulações em computador, baseando-se em diversas equações matemáticas e dados levantados no campo. Os resultados mostraram que a aplicação manual apresentou-se mais econômica apenas para trechos com baixas infestações (até $10 \%$, em média). Na composição de custos, os gastos com herbicidas corresponderam a cerca de $80 \%$ do total para a aplicação mecanizada e apenas $8 \%$ na manual. Levando-se em conta os fatores operacionais e de mão-de-obra, em apenas um dos trechos avaliados houve vantagem econômica do uso da aplicação manual.

Palavras chave: pulverização, custos, área não agrícola.

\section{ABSTRACT}

\section{Economical viability of manual and mechanized application of herbicides in railways}

Mechan ized and manual sy st ems of herbicide application in railways present either advantages or disadvantages. The main advantage of mechanized application is related to its wide operational capacity which covers large distances in short time. However, in function of the equipment characteristics, an execessive waste of herbicide usually occurs. The main advantage of manual application is the direct spraying in the infested area, decreasing the quantity of herbicide applied. On the other hand, its reduced operational capacity delays the application of the large areas, becoming the operation dependent on climatic and operational problems of the railway. The aim of this work was to perform a comparative analy sis of operational and economical characteristics of the systems, pointing out the most important fact or to be evaluated for its implantation. Therefore, simulations we re performed by computer, considering several mathematical equations and data obtained in field. The results showed that manual ap plication was more

1 Recebido para publicação em 28/07/95 e na forma revisada em 21/05/96.

2 Prof. Assistente Doutor. Depto. Eng. Rural, FCA-UNESP, Caixa Postal 237, CEP 18.603-970, Botucatu/SP, Brasil.

3 Prof. Assistente Doutor, Depto. Agricultura e Melhoramento Vegetal. FCA-UNESP.

4 Eng. Agr., Monsanto do Brasil Ltda., Rua Paes Leme. 504, CEP 05.424-000, São PaulolSP. 
economical only for areas with low infestations. Concerning costs, the expenses with herbicides corresponded to approximately $80 \%$ of the total for mechanized application, and $8 \%$ for manual application. By considering operational and labor

\section{INTRODUÇÃO}

O controle das plantas daninhas é de grande importância dentro da manutenção das áreas de ferrovia. A ausência de controle da infestação pode prejudicar as atividades relaciona das ao tráfego de trens e a manutençã o das linhas, pátios e obras de engenharia. Das modalidades de controle mais comumente utilizadas, destaca-se o controle químico, ou seja, a aplicação de herbicidas.

Diversos estudos têm sido realizados com o intuito de desenvolver e adaptar metodologias para a aplicação e avaliação do uso de herbicidas em áreas ferroviárias, podendo-se destacar, recentemente, os trabalhos de Furlani Junior et al. (1992a,b), Antuniassi \& Velini (1992) e Velini \& Antuniassi (1992). Grande parte destes estudos concentram-se na utilização de equipamentos mecanizados para a pulverização. Entretanto, em função das peculiaridades de cada empresa ou mesmo de cada trecho de ferrovia, a utilização de equipamentos manuais vem sendo estudada como uma alternativa aos equipamentos tradicionais.

Do ponto de vista operacional, o sistema mecanizado destaca-se pela grande capacidade operacional, cobrindo trechos extensos em curtos espaços de tempo. Entretanto, em função das características dos equipamentos utilizados, mu it as vezes ocorre grande desperdício de herbicidas nas aplicações pós-emergentes. Em locais onde a infestação não se apresenta uniforme e contínua, a grande velocidade de desloc amento do equipamento dificulta a ação do operador na abertura e fechamento das barras de pulverização. Desta forma, erros podem ocorrer levando fact or, in only one of the areas evaluated, economical advantage in the usage of manual application was presented.

Key words: Application methods, costs,

herbicida a áreas não infestadas ou ocasionando fal has na cobertura da vegetação existente. Tal fato acaba por influenciar o operador a realizar a pulverização em área total, aumentando ainda mais o desperdício de herbicida.

A aplicação manual, através de pulverizadores costais, tem como principal carac terís tic a a pos sibilidade da pulverização dirigida às áreas infestad as, diminu in do as quantidades de herbicida aplicado. Por outro lado, sua reduzida capacidade operacional faz com que a aplicação de longos trechos seja demorada, sujei tando-se aos problemas climáticos e operacionais na ferrovia. Outro fator importante a ser considerado é o controle ambiental. É notório que as aplicações mecanizadas têm sido realizadas com um correto e eficiente controle dos riscos de con tami nação do ambiente. En tret anto, a aplicação manual, por envolver menores quantidades de defensivos, deve ser considerada como de menor risco ambiental. Ressalta-se, porém, que independentemente da forma de ap licação, o uso de he rbicidas em áreas de ferrovias apresenta, atualmente, um risco reduzido de danos ao ambiente.

Partindo-se deste princípio, a decisão do uso de uma ou outra forma de pulverização pode ser tomada em função de uma análise econômica de cada caso. Antunias si \& Velini (1992) realizaram estudos operacionais e econômicos da aplicação mecanizada de herbicidas em ferrovias. Foram determinados os custos de diferentes sistemas de controle, utilizando-se dive rsos herbicidas. De acordo com os autores, para as condições de trabalho na FEPASA (Ferrovia Paulista S.A.), o sistema mais econômico envolve aplicações seqüenciais do herbicida glyphosate.

Planta Daninha, v. 14, n. 1, 1996. 
O objetivo deste trabalho foi realizar urna análise comparativa das características operacionais e econômicas dos sistemas mecanizado e manual de aplicação de herbicidas pós-emergentes em áreas de ferrovias, apontando os fatores mais importantes a serem avaliados para a sua implantação. Para tanto, foram realizadas simulações em computad or, ba seando-se em diversas equações matemáticas e dados levantados no campo.

\section{MATERIAL E MÉTODOS}

\section{Sistemas de aplicação de herbicidas}

Para a realização deste trabalho foram an al isa do s do is si stemas de aplicação de herbicidas em ferrovias: um com aplicação mecanizada e outro com aplicação manual, através de pulverizadores manuais costais. Ambos os sistemas consideraram as condições ope racionais e econômicas ex istentes na Companhia Vale do Rio Doce (CVRD) - Estrada de Ferro Vitória-Minas (EFVM).

\section{Sistema mecanizado}

O si st ema mecanizado de ap li cação consiste numa composição com locomotiva e quatro vagões (aplicador, reservatório de água, oficina e dormitório/restaurante). Os conjuntos de bombeamento e as barras com bicos pulverizad or es possibilitam velocidades de deslocamento de 12,5 e $25 \mathrm{~km} / \mathrm{h}$ com faixas aplicadas entre 1,1 e 7,4 m e vol ume de calda ap licado por hectare de até 4600 litros. A capacidade total dos reservatórios de água e calda soma m 45000 litros. São utilizados seis trabalhadores por turno de serviço.

\section{Sistema manual}

O sistema manual de aplicação é composto por um grupo de três aplicadores munidos de pulverizadores costais com capacidade de 20 litros, sendo do is destes equipados com barra de pulverização para $1,5 \mathrm{~m}$ de faixa e um com apenas 1 bico para faixa de $0,5 \mathrm{~m}$. Acompanham um veículo para transporte e dois trabalhadores para o apoio.

\section{Avaliação das condições de infestação}

Os dados relativos à infestação por plantas daninhas nos trechos de ferrovia foram obtidos através de levantamentos junto à $C V R D-E F V M$ (Tabela 1). Quantificaram-se as porcentagens de cobertura do solo por plantas daninhas no lastro (área de pedras onde são assentados os trilhos) e nas banquetas (faixas de terra laterais ao lastro).

TABELA 1 - Locais de avaliação da infestação e porcentagem de cobertura do solo por plantas daninhas. Dados obtidos em fevereiro de 1992.

\begin{tabular}{|c|c|c|c|c|c|}
\hline $\begin{array}{c}\text { Trecho } \\
\text { avaliado }\end{array}$ & Regional & Distrito & Local & $\begin{array}{c}\% \text { de cobertura } \\
\text { no lastro } \\
\end{array}$ & $\begin{array}{c}\% \text { de cobertura na } \\
\text { banqueta }\end{array}$ \\
\hline 1 & $1^{2}$ & $1^{\circ}$ & $\mathrm{km} 000$ a 102 & 1 & 45 \\
\hline 2 & $1^{3}$ & $2^{\circ}$ & km 102 a 202 & 2 & 75 \\
\hline 3 & $2^{*}$ & $3^{\circ}$ & $\mathrm{km} 202$ a 325 & 2 & 75 \\
\hline 4 & $2^{s}$ & $4^{\circ}$ & $\mathrm{km} 325$ a 438 & 1 & 35 \\
\hline 5 & $3^{a}$ & $5^{\circ}$ & $\mathrm{km} 438$ a 542 & 1 & 35 \\
\hline 6 & $3^{a}$ & $6^{\circ}$ & C. Lacerda/Fábrica & 1 & 35 \\
\hline 7 & $4^{3}$ & $7^{\circ}$ & km 062 a 169 & 1 & 60 \\
\hline 8 & $4^{a}$ & $8^{\circ}$ & C. Lacerda/B. Cocais & 1 & 100 \\
\hline 9 & $4^{a}$ & $8^{\circ}$ & B. Cocais/C. Eduardo & 0 & 2 \\
\hline
\end{tabular}




\section{Levantamento dos parâmetros operacionais e econômicos}

Para a realização das análises operacionais e econômicas foram utilizadas informações de custos e desempenho das operações relativas às condições da $C V R D-E F V M$. Os Parâme tros iniciais utiliza dos (fornecidos pela $C V R D-E F V M$ ou calculados a partir de tais informações) estão listados na Tabela 2.

TABELA 2 - Parâmetros iniciais utilizados para as simulações operacionais e econômicas.

\begin{tabular}{|c|c|c|}
\hline \multirow{2}{*}{$\begin{array}{l}\text { Parâmetros } \\
\text { Dados gerais: }\end{array}$} & \multicolumn{2}{|c|}{ Valores } \\
\hline & & \\
\hline Largura média da banqueta & 1,5 & $\mathrm{~m}$ \\
\hline Largura média do lastro & 8,0 & $\mathrm{~m}$ \\
\hline Preço do herbicida (glyphosate - $360 \mathrm{~g}$ i.a. $/ \ell$ ) & 10,00 & USS/litro \\
\hline Dose do herbicida na banqueta & 6,0 & $\ell /$ ha \\
\hline Dose do herbicida no lastro & 6,0 & $\ell /$ ha \\
\hline Jornada diária de trabalho & 8 & $\mathrm{~h}$ \\
\hline \multicolumn{3}{|l|}{ Salários (Valores referentes a CVRD-EFVM): } \\
\hline Ajudantes & 9,00 & US\$/hora \\
\hline Técnicos & 11,00 & US\$/hora \\
\hline Maquinistas & 17,00 & US\$/hora \\
\hline \multicolumn{3}{|l|}{ Salários (Valor referente a empreiteiras contratadas): } \\
\hline Ajudantes & 5,00 & US\$/hora \\
\hline \multicolumn{3}{|l|}{ Sistema manual: } \\
\hline Velocidade de aplicação & 4,1 & $\mathrm{~km} / \mathrm{h}$ \\
\hline Fator de eficiência & 0,25 & \\
\hline Faixa de aplicação total & 3,5 & $\mathrm{~m}$ \\
\hline \multicolumn{3}{|l|}{ Mão-de-obra empregada: } \\
\hline Ajudantes & 3 & \\
\hline Técnicos & 1 & \\
\hline Maquinistas & 1 & \\
\hline Custo dos equipamentos de aplicação & 0,05 & US\$/hora \\
\hline Custo dos equipamentos de transporte & 20,00 & US\$/hora \\
\hline Tempo diário de uso dos equipamentos manuais costais & 8 & horas \\
\hline Tempo diário de uso do transporte & 2 & horas \\
\hline \multicolumn{3}{|l|}{ Sistema mecanizado: } \\
\hline Velocidade de aplicação & 25 & $\mathrm{~km} / \mathrm{h}$ \\
\hline Fator de eficiência & 0,8 & \\
\hline Faixa de aplicação total (linha dupla) & 11 & $\mathrm{~m}$ \\
\hline \multicolumn{3}{|l|}{ Mão-de-obra empregada: } \\
\hline Ajudantes & 4 & \\
\hline Técnicos & 1 & \\
\hline Maquinistas & 1 & \\
\hline Custo do equipamento de aplicação & 28,07 & US\$/hora \\
\hline Tempo diário de uso do equipamento de aplicação & 4 & horas \\
\hline
\end{tabular}


Simulação da utilização dos sistemas

As análises comparativas foram realizadas através de simulações do uso dos sistemas nos tre chos avaliados, utilizando -se uma planilha eletrônica em computador compatível com IBMPC (programa Lotus $123^{\circledR}$ ). Para a elaboração da planilha foram utilizadas equações matemáticas de uso consagrado no cálculo operacional e econômico de máquinas e equipamentos (Balastreire, 1987; Mialhe, 1974) e ta mbém equações deduzidas em função de situações práticas. Os procedimentos adotados foram semelhantes aos descritos por Antuniassi (1993). A listagem do conteúdo das células da planilha de simulação, mostrando as diversas equações, é apresentada na Tabela 3. Para tornar possível a comparação prática dos sistemas, em função do grande número e diversidade das variáveis envolvidas, adotou-se como princípio que a apl ica ção mecani zada oco rre sempre em áre a total, independentemente do nível de infestação da linha. No caso do sistema manual, considerou-se que a aplicação ocorre somente sobre a vegetação existente.

Para facilitar a visualização dos resultados foi definido o índice de viabilidade econômica (I), que consiste na divisão do custo total do sistema mecanizado pelo custo total do sistema manual. Desta forma, quando I> 1 , existe vantagem no uso do sistema manual; $\mathrm{I}<1$ indica melhor resultado econômico no uso do sistema me ca ni za do e I = 1 id en tifica si tuações de igualdade de custos entre os sistemas.

TABELA 3 - Listagem do conteúdo das células da planilha de simulação

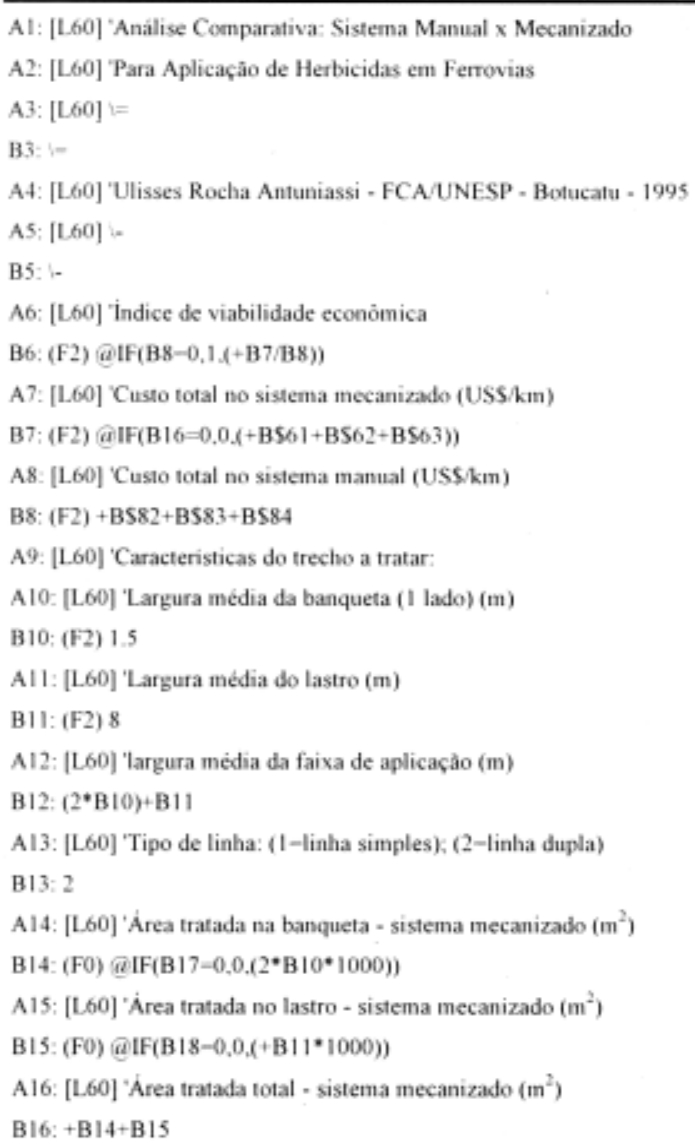

A 17: [L60] 'Cobertura do solo por plantas daninhas na banqueta (\%) B17: (F2) 10.17

A 18: [L 60$]$ 'Cobertura do solo por plantas daninhas no lastro (\%) B18: (F2) 10.17

A 19: [L60] "Área tratada na banqueta - sistema manual $\left(\mathrm{m}^{2}\right)$ B 19: $(\mathrm{F} 0)+\mathrm{B} 17 * 2 * \mathrm{~B} 10 * 10$

A20: [L60] 'Área tratada no lastro - sistema manual $\left(\mathrm{m}^{2}\right)$

B20: $(\mathrm{F} 0)+\mathrm{B} 18 * \mathrm{~B} 11 * 10$

A21: [L60] 'Preço do herbicida (USS/])

B21: (F2) 10

A22: [L.60] 'Dose do herbicida na banqueta ( $\delta$ ha)

B22: (F2) 6

A23: [1.60]'Dose do herbicida no lastro ( $f / \mathrm{ha}$ )

B23: (F2) 6

A24: [L60] 'Herbicida aplicado na banqueta - sistema mecanizado $(1 / \mathrm{km})$ B24: (F2) +B14*B22/10000

A25: [L60] 'Herbicida aplicado no lastro - sistema mecanizado ( $1 / \mathrm{km})$

B25: $(\mathrm{F} 2)+B 15^{*} \mathrm{~B} 23 / 10000$

A26: [L60] Herbicida aplicado na banqueta - sistema manual (1/ km)

B26: (F2) +B19*B22/10000

A27: [L60] Herbicida aplicado no lastro - sistema manual $(\mathrm{L} / \mathrm{km})$

B27: (F2) +B20*B23/10000

A28: [L60] 'Caracteristicas operacionais:

A29: [L.60)] 'Velocidade de aplicação no sistema manual $(\mathrm{km} / \mathrm{h})$

B29: (F2) 4.I

A30: [L60] 'Fator de eficiència para aplicaçào manual

B30: (F2) 0.25 
TABELA 3 - Continuação.

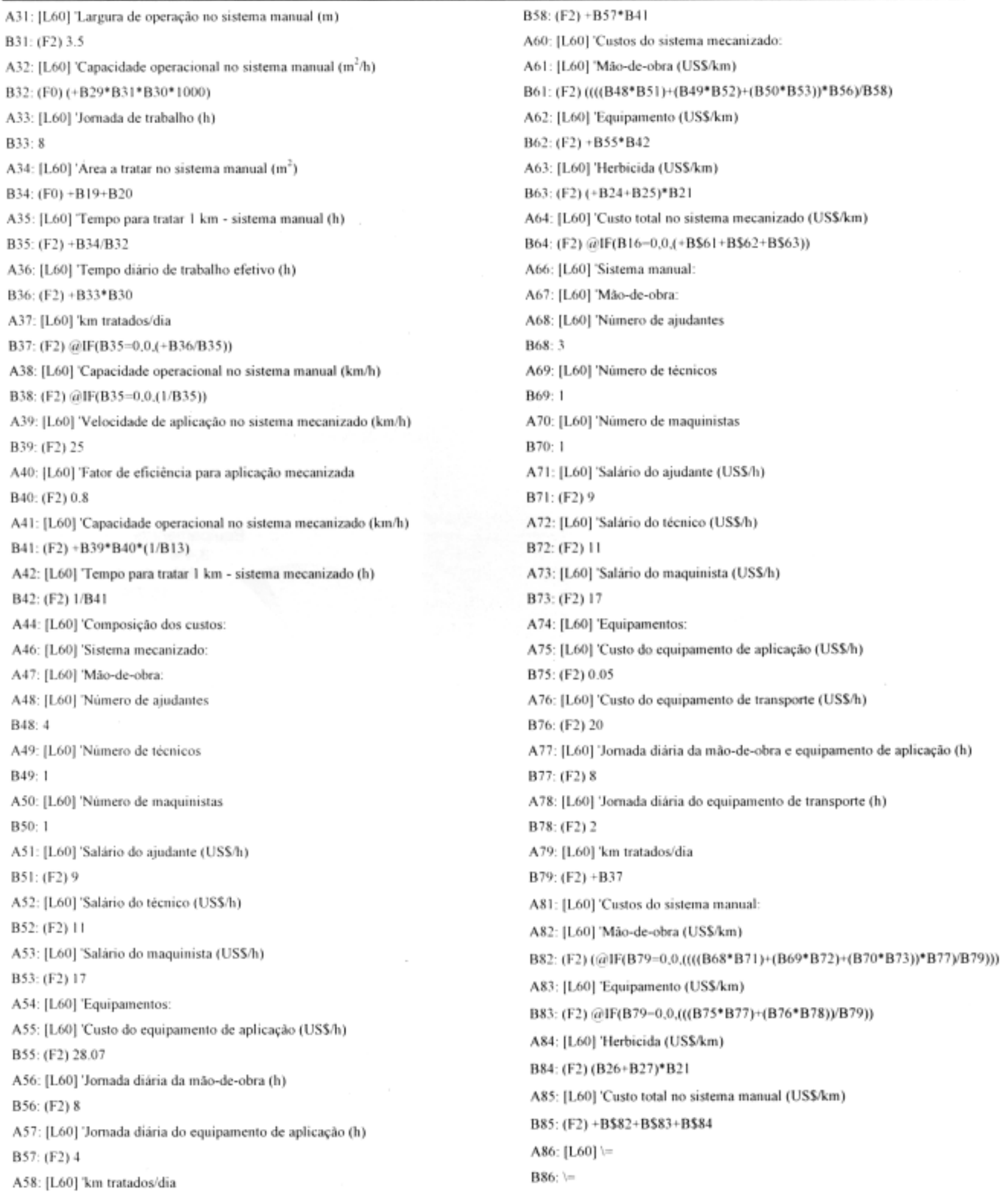

Planta Daninha, v. 14, n. 1, 1996. 


\section{RESULTADOS E DISCUSSÃO}

\section{Composição dos custos dos sistemas}

A Figura 1 mostra a composição percentual média dos custos referentes aos sistemas manual e mecanizado de aplicação de herbicidas em ferrovias. Observa-se que no sistema manual a maior parcela do custo refere-se à mão-de-obra utilizada, sendo que herbicidas e equipamentos têm porcentagens muito menores e se me lhantes entre si. Tal fato de monstra a importância do investimento no treinamento e melhoria da qualidade da mão-de-obra, como fator de aumento de produtividade e redução de custos. Outra possibilidade para aumentar a viabilidade econômica do sistema é a utilização de serviços de terceiros (empreiteiras), cujos custos de mão-de- obra são geralmente menores do que os das empresas ferroviárias.

Para o sistema mecanizado, a maior parcela do custo refere-se ao gasto com herbicidas. Neste caso, todo empenho deve ser direcionado à redução das quantidades necessárias deste fator. A contenção das despesas passa obrigatoriamente por uma racional ização da operação de aplicação, procurando-se diminuir os er ros e mi ni mi zando -se o de sperdício do defensivo. Quanto aos equipamentos, observa-se que, apes ar de serem utilizados apl icadores de grande porte, sua participação no custo total do sistema é reduzida. Vale ressaltar, ainda, que com relação ao sistema mecanizado, tais observações coincidem com os resultad os descritos por Antuniassi \& Velini (1992).

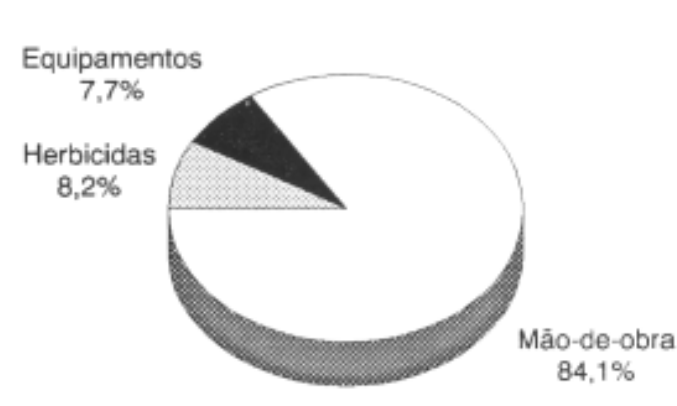

Sistema manual

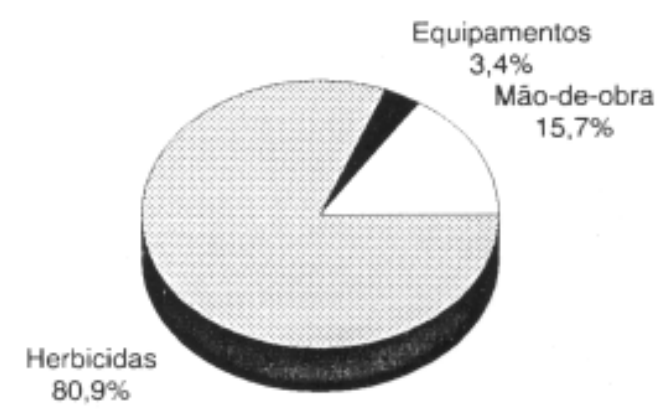

Sistema mecanizado

FIGURA 1 - Composição percentual dos custos relativos aos sistemas manual e mecanizado de aplicação de herbicidas em ferrovias. Valores obtidos considerando-se os custos da mão-de-obra na CVRD-EFVM.

\section{Análise da viabilidade econômica}

A Figura 2 apresenta os resultados da simulação do uso dos sistemas nos trechos onde foi realizado o levantamento da infestação por plantas daninhas. Observa-se que o custo da aplicação mecanizada foi o mesmo para os trechos 1 a 8 , independentemente das diferenças nos níveis de infestação. Tal fato ocorre em função da pulverização ser realizada em área total, face às dificuldades já expostas anteriormente. Somente no trecho 9 foi possível a particularização da aplicação, onde a infestação é nula no lastro. Para este local foram contabilizados apenas os custos da pulverização na banqueta. Os custos no sistema manual foram proporcionais às porcentagens de infestação das áreas aval iadas. Ou tro fator analisado foi o índice de viabilidade econômica

Planta Daninha, v. 14, n. 1, 1996. 
(I) para os diversos trechos. Observa-se que, para a situação em que se encontravam os locais na época da avaliação, somente ocorre vantagem no uso do sistema manual no trecho 9. Entretanto, quando se utilizam, para o cálculo dos custos, os valores da mão-de-obra referentes às empreiteiras, tal viabilidade se estende aos trechos 4,5 e 6 (Figura 3).

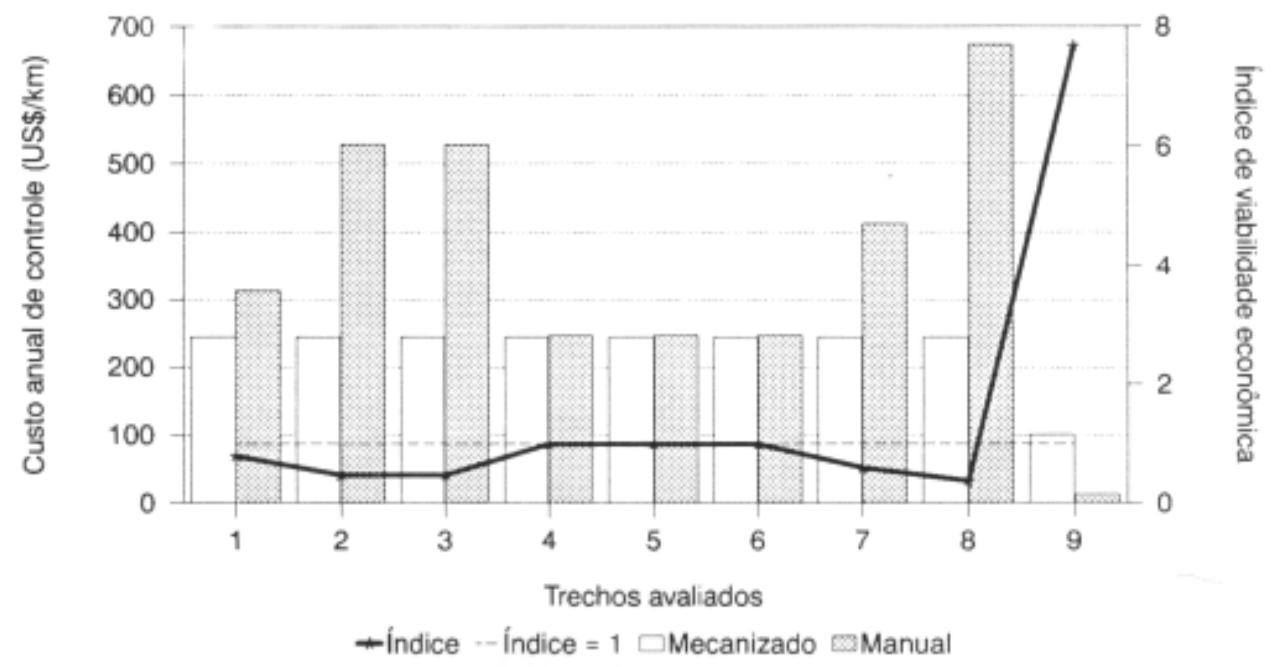

FIGURA 2 - Custo anual de controle das plantas daninhas e índice de viabilidade económica (I) para os trechos avaliados na $C V R D-E F V M$, considerando mão-de-obra da própria ferrovia.

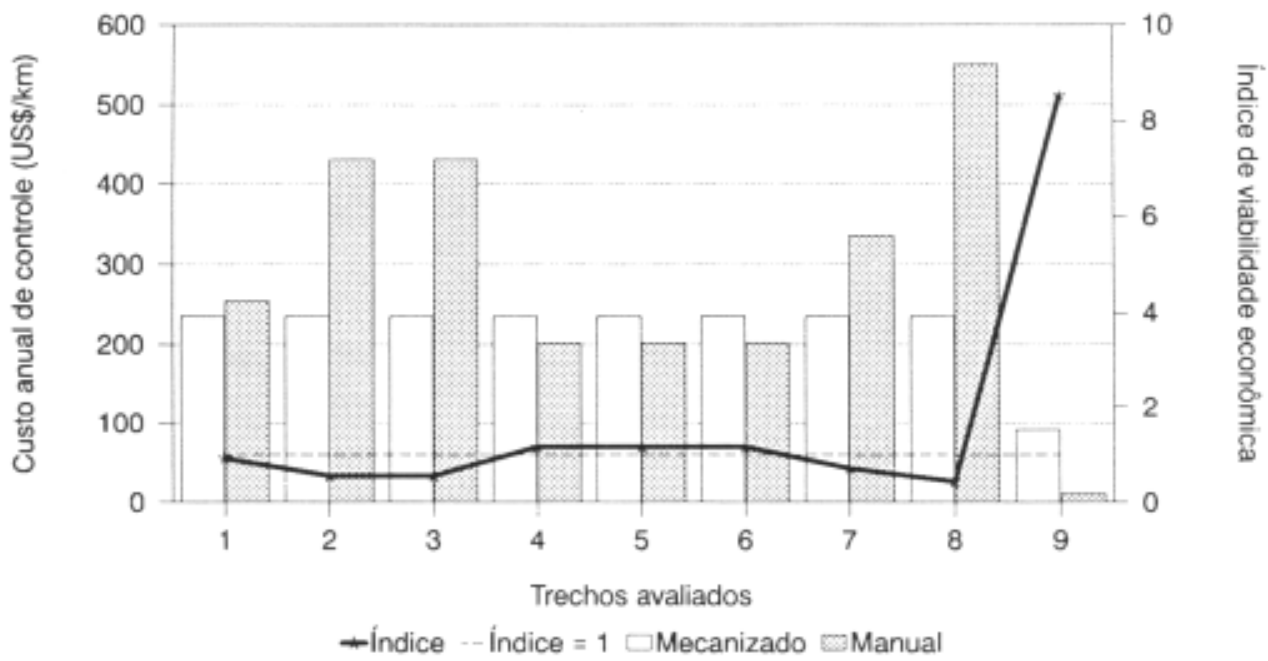

FIGURA 3 - Custo anual de controle das plantas daninhas e índice de viabilidade econômica (I) para os trechos avaliados na $C V R D-E F V M$, considerando mão-de-obra de empreiteiras contratadas.

Analisando-se o comportamento do $\mathbf{I}$ em função da variação da infestação média (Figura 4), observa-se que, para o uso de mão-de-obra própria, somente ocorre vantagem no uso do sistema manual para casos de infestações muito baixas (até 10\%). Mes mo considerando-se os custos de mão-de-obra das empreiteiras, tal valor permanece baix o (12\%). Is to indica que a 
aplicação manual só será uma opção efetivamente econômica após a consolidação completa dos programas de controle das plantasdaninhas, já que o uso constante de tais práticas provoca uma red ução da infestação mé dia ao lon go do tempo.
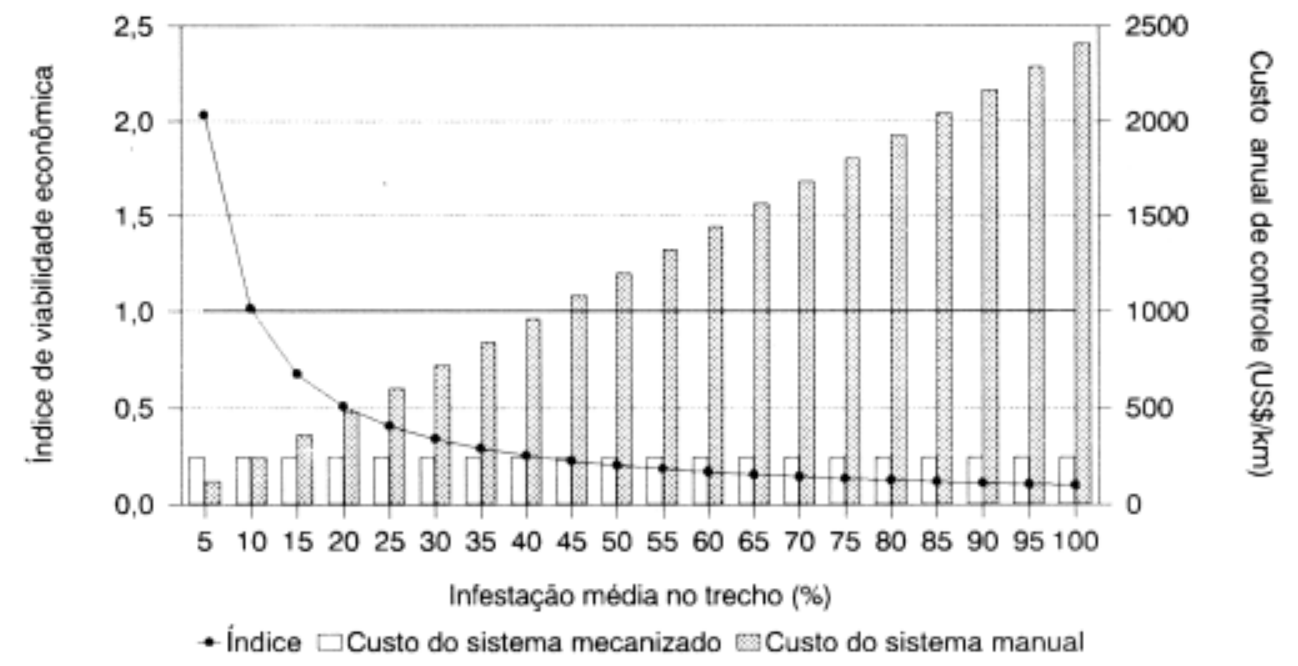

FIGURA 4 - Custo anual de controle das plantas daninhas e índice de viabilidade economica (I) considerando-se uma variação da infestação média entre 5 e 100\%. Valores relativos ao uso de mão-de-obra própria.

Out ro pon to importante a ser observado é a re lação entre o aumento da infe st ação e o aumento do custo no sistema manual. Tal comportamento pode ser creditado a dois fatores principais. Inicialmente, como a aplic ação manual é realizad a somente sobre a área efetivamente infestada, fica evidente a relação entre o custo total e o nível de infestação. Isto não ocorre no sistema mecanizado, onde a aplicação é realizada em área total. Posteriormente, a diferença entre as capacidades operacionais dos sistemas influencia diretamente os custos finais obtidos. Como a capacidade operacional média do sistema manual é muito baixa, quando comparada a do mecanizado $(0,358 \mathrm{ha} / \mathrm{h}$ no manual e $11 \mathrm{ha} / \mathrm{h}$ no mecanizado), em áreas de grande infestação sua realização é morosa. Assim, o custo da mão-de-obra se torna elevado, em função dos salários serem calculados pelas horas trabalhadas.

As Figuras 5 e 6 mostram análises da sensibilidade do índice de viabilidade econômica (I) em relação à variação dos principais fatores en volvidos na determinação dos custos dos sistemas, seguindo metodologia descrita por Antuniassi (1993). Nestas análises, os fatores tiveram seus valores básicos variados entre $+50 \%$ e - 50\%; desta forma, usando como exemplo o preço do herbicida (US\$ 10,00/e p.c.), seu valor foi simulado entre US\$ $5.00(-50 \%) \mathrm{e}$ US $\$ 15,00(+50 \%)$. Procedendo - se desta maneira, e mantendo-se fixos os demais fatores envolvidos, calculou-se o I para tal situ ação. Com este procedimento, o ângulo médio de inclinação das curvas com relação ao eixo "x" fornece uma idéia da sensibilidade de I com rel ação ao fat or em questão. Assim, um ângulo maior mostra uma sensibilidade superior e viceversa. Este tipo de análise permite a visualização mais efetiva da importância relativa entre os fatores que influenciam a composição dos custos dos sistemas. 


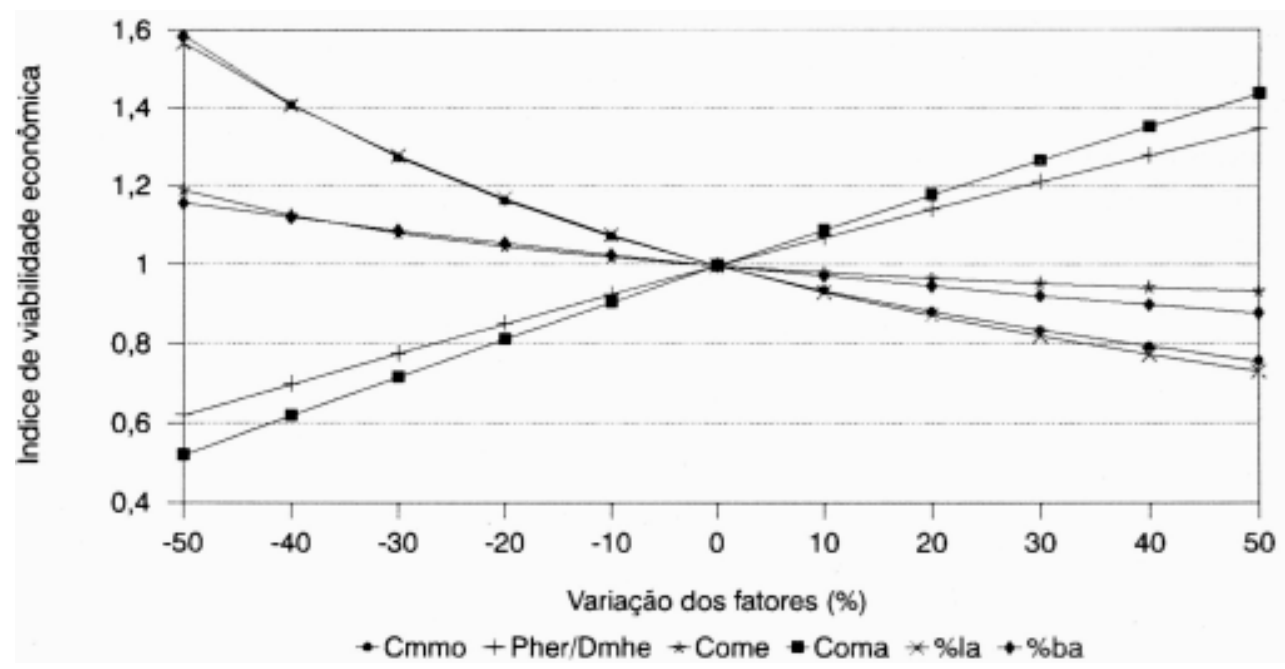

FIGURA 5 - Variação do índice de viabilidade econômica (I) em função da vari ação dos seguintes fat ore s: custo médio da mão-de-obra ( $\mathrm{Cmmo})$, preço ou dose média do herbicida (Pher/Dmhe), capacidade operacional do sistema mecanizado (Come), capacidade operacional do sistema manual (Coma), porcentagem de cobertura do solo por plantas daninhas no lastro (\%la) e porcentagem de cobertura do solo por plantas daninhas na banqueta(\%ba). Simulação baseada em uma infestação média da ordem de $10 \%$ de cobertura do solo por plantas daninhas.

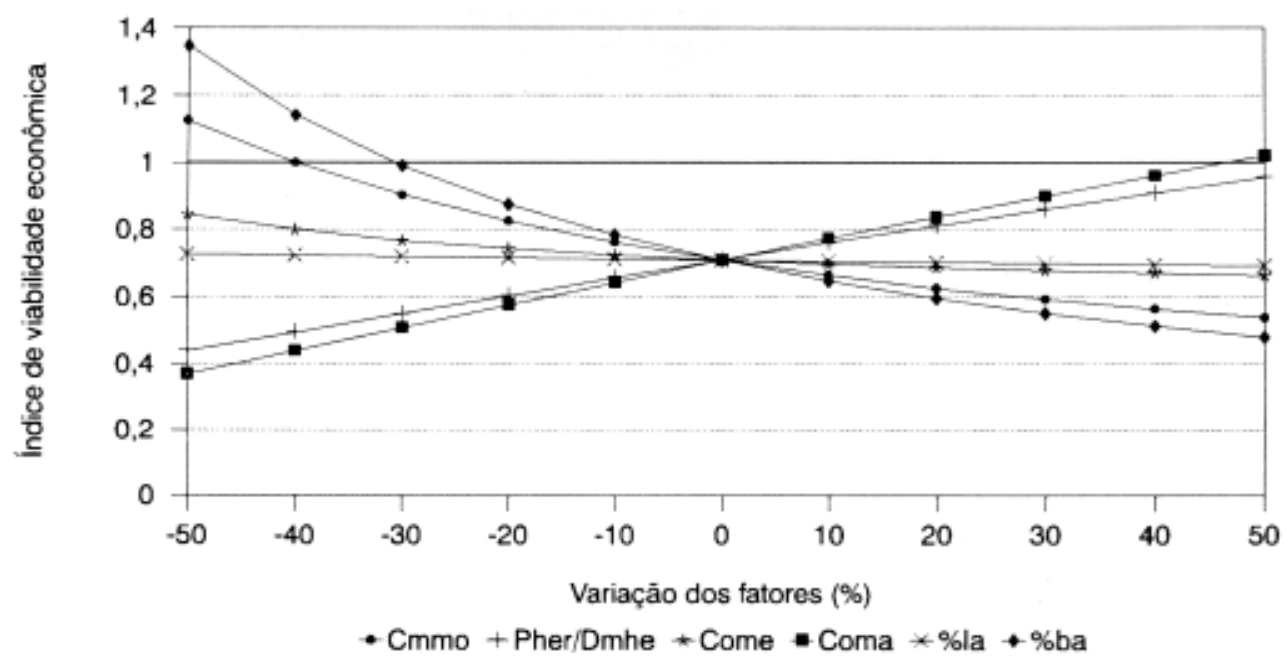

FIGURA 6 - Variação do índice de viabilidade econômica (I) em função da vari ação dos seguintes fatores: cus to mé dio da mão-de-obra ( $\mathrm{Cmmo})$, preço ou dose média do herbicida (Pher/Dmhe), capacidade operacional do sistema mecanizado (Come), capacidade operacional do sistema manual (Coma), porcentagem de cobertura do solo por plantas daninhas no lastro (\%la) e porcentagem de cobertura do solo por plantas daninhas na banqueta(\%ba). Simulação baseada na cobertura média do solo por plantas daninhas nos trechos avaliados na CVRD-EFVM. 
A análise apresentada na Figura 5 partiu do princípio de que a infestação média do local em questão permitia um $\mathbf{I}=\mathbf{1}$ (igual viabilidade econômica de ambos os sistemas). Pode-se observar, deste modo, que a capacidade operacional do sistema manual e o preço/dose do herbicida são fatores diretamente proporcionais ao I, ou seja, acréscimos nos valores destes fatores provocam aumentos no valor de I, tornando o sistema manual mais viável. O inverso também é verdadeiro. Situação oposta ocorre com os demais fatores avaliados (custo médio da mão-de-obra, capacidade operacional do sistema mecanizado e porcentagens de cobertura do solo por plantas daninhas no lastro e banqueta). Pode-se destacar, ai nda, a grande importância da ca pacidade operacional do sistema manual, do custo médio da mão-de-obra e da infestação no lastro na formação do índice de viabilidade económica (I).

$\mathrm{Na}$ Figura 6 o ponto de partida foi o valor da infestação média dos trechos avaliados na $C V R D$ EFVM, procurando, desta forma, representar uma média das condições de viabilidade dos sistemas na Companhia. Observase, inicialmente, que o I parte de um valor próximo a 0,7 , indicando melh or resultado econômico no sistema mecanizado. Analisando-se o comportamento dos fatores, verifica-se que os únicos capazes de reverter a situação, dentro das faixas de variação estudadas, são o custo médio da mão-de-obra, infestação na banqueta e capacidade operacional do sistema manual. Nota-se, por exemplo, que reduções da ordem de $30 \%$ na infestação da banqueta ou reduções de $40 \%$ do custo da mão-de-obra podem tornar viável o uso do sistema manual. Vale ressaltar, entretanto, que as análises realizadas nas Figuras 5 e 6 levaram em consideração o custo da mão-de-obra da $C V R D$ EFVM. Para efeito comparativo, quando tal fator foi substituído pelo valor referente às empreiteiras, o I médio da companhia ficou próx imo a 0,8 , não alterando, portanto, a vantagem no uso do sistema mecanizado.
Assim, o modelo mate mático proposto, bem como os resultados e as análises apresentadas são válidos para situações de aplicação de herbicidas em ferrovias, resguardando-se os parâmetros e as restrições inicialmente propostas. Entretanto, através de algumas modificações, é possível adaptar o modelo para as mais diversas condições de aplicação de defensivos, inclusive em áreas de produção agrícola.

\section{AGRADECIMENTOS}

Agradecemos a Companhia Vale do Rio Doce (CVRD) - Est'ada de Ferro Vilória-Minas (EFVM), Departamento de Meio Ambiente, pelo fornecimento das informações necessárias à realização deste trabalho.

\section{LITERATURA CITADA}

ANTUNIASSI, U.R. Simulação operacional e econômica e desenvolvimento da cultura do milho (Zea mays L.) semeado em janeiro, em função de dois tipos de preparo do solo e cinco modalidades de controle de plantas invaso ras. Botucatu: UNESP, 1993. $141 \mathrm{p}$. Tese (Doutorado em Energia na Agricultura).

ANTUNIASSI, U.R., VELINI, E.D. Avaliação econômica e operacional de diferentes sistemas de controle químico de plantas daninhas em ferrovias. In: CONGRESSO BRASILEIRO DE ENGENHARIA AGRÍCOLA, 21, 1992, Santa Maria. Anais... Santa Maria: Departamento de Engenharia Rural, Universidade Federal de Santa Maria, 1992. p.2082-2096.

BALA STREIRE, L.A. Máqui nas agrícola s. São Paulo: Manole, 1987.307p.

Planta Daninha, v. 14, n. 1, 1996. 
FURLANI JUNIOR, J.A., NOGUEIRA, H.C., ANTUNIASSI, U.R. Construção de protótipo de aplicador de herbicidas em ferrovias. In: CONGRESSO BRASILEIRO DE ENGENHARIA AGRÍCOLA, 21, 1992, Santa Maria. Anais... Santa Maria: Departamento de Engenharia Rural, Universidade Federal de Santa Maria, 1992a. p.2026-2034.

FURLANI JUNIOR, J.A., NOGUEIRA, H.C., ANTUNIASSI, U.R. Estudo comparativo de parâmetros operacionais e econômicos entre a capina química e manual em ferrovias. In: CONGRESSO BRASILEIRO DE ENGENHARIA AGRÍCOLA, 21, 1992, Santa Maria. Anais... Santa Maria:
Departamento de Engenharia Rural, Universidade Federal de Santa Maria, 1992b. p.2015-2025.

\section{MIALHE, L.G. Manual de mecanização} agrícola. São Paulo: Ceres, 1974. 301p.

VELINI, E.D., ANTUNIASSI, U.R. Avaliação comparativa de sistemas de controle químico de plantas daninhas em ferrovias. In: CONGRESSO BRASILEIRO DE ENGENHARIA AGRÍCOLA, 21, 1992,

Santa Maria. Anais... Santa Maria: Departamento de Engenharia Rural, Universidade Federal de Santa Maria, 1992. p.2048-2057. 